\title{
Ins and Outs of Cerebellar Modules
}

\author{
Tom J. H. Ruigrok
}

Published online: 16 March 2010

(C) The Author(s) 2010. This article is published with open access at Springerlink.com

\begin{abstract}
The modular concept of cerebellar connections has been advocated in the lifetime work of Jan Voogd. In this concept, a cerebellar module is defined as the conglomerate of one or multiple and non-adjacent, parasagittally arranged zones of Purkinje cells, their specific projection to a well-defined region of the cerebellar nuclei, and the climbing fiber input to these zones by a welldefined region of the inferior olivary complex. The modular organization of these olivo-cortico-nuclear connections is further exemplified by matching reciprocal connections between inferior olive and cerebellar nuclei. Because the different regions of the cerebellar nuclei show highly specific output patterns, cerebellar modules have been suggested to constitute functional entities. This idea is strengthened by the observation that anatomically defined modules adhere to the distribution of chemical markers in the cerebellar cortex suggesting that modules not only differ in their input and output relations but also may differ in operational capabilities. Here, I will briefly review some recent data on the establishment of cerebellar modules in rats. Furthermore, some evidence will be shown suggesting that the other main afferent system (i.e., mossy fibers), at least to some extent, also adheres to the modular organization. Finally, using retrograde transneuronal tracing with rabies virus, some evidence will be provided that several cerebellar modules may be involved in the control of individual muscles.
\end{abstract}

Keywords Cerebellar modules · Connections · Purkinje cells $\cdot$ Climbing fibers $\cdot$ Mossy fibers $\cdot$ Rabies tracing

\footnotetext{
T. J. H. Ruigrok $(\bowtie)$

Department of Neuroscience, Erasmus MC Rotterdam, P.O. Box 2040, 3000 CA Rotterdam, The Netherlands e-mail: t.ruigrok@erasmusmc.nl
}

\section{Introduction}

It is well-known that cerebellar connections generally adhere to longitudinal patterns [1-4]. These patterns are recognized in the climbing fiber projections of the inferior olive to the cerebellar cortex [5-8] as well as in the projections of the Purkinje cells to the cerebellar and vestibular nuclei [9-11]. Moreover, the olivo-corticonuclear organization is nicely matched by the pattern of climbing fiber collaterals to the cerebellar nuclei and the GABAergic projection from the cerebellar nuclei to the inferior olive as indicated schematically in Fig. 1 [12-15]. The output of a module is directed to a specific combination of brainstem and thalamic nuclei [16, 17].

Physiological studies have demonstrated that sensory information from the body reaches the inferior olive by way of several spino-(bulbo-)-olivary pathways which link perfectly with the organization of the olivo-cerebellar pathways [18-21]. Interestingly, although it was generally assumed that the operational characteristics were similar all over the cerebellar cortex, recent discoveries have shown that many biochemical markers, such as $5^{\prime}$ nucleotidase, aldolase $C$, phospholipase $C \beta 3$ and $\beta 4$, excitatory amino acid transporter 4 , and metabotropic glutamate receptor 1a are heterogeneously distributed, usually in parasagittally organized bands that correspond to the organization of the olivo-cortico-nuclear projection (for review see [2]).

Here, we review some of our data on the establishment of the modular organization of cerebellar connections. In addition, some experiments are described which suggest that the organization of mossy fiber projections, at least to some extent, adheres to the modular organization. Furthermore, we show that the output of several modules is used to control the activity of individual muscles. 
a

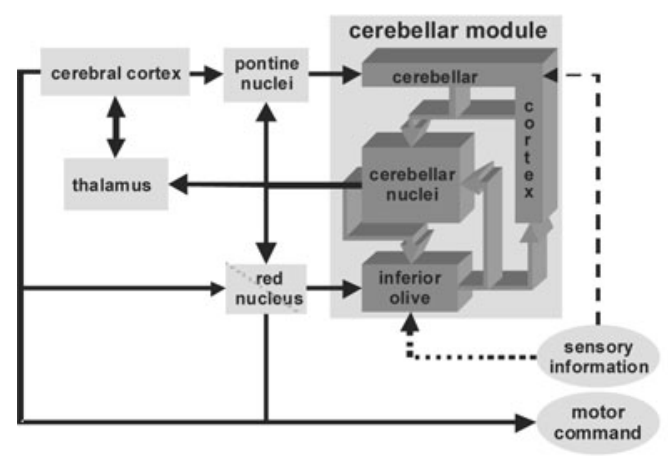

Fig. 1. Schematic diagrams of cerebellar modules. a Basic diagram of a generalized cerebellar module and its connections. A strip of Purkinje cells receives input from a selected area of the inferior olive and projects to a specific region of the cerebellar nuclei. Reciprocal connections between olive and nuclei complete the module. The output of a module is directed to premotor areas in the brainstem as well as to the thalamus. In addition, feedback is provided to

\section{Methods}

\section{Animals}

Experiments were performed on either male Wistar rats of $250-350 \mathrm{~g}$ or adult male C57BL/6 mice. All experiments were carried out in accordance with NIH guidelines for the care and use of laboratory animals and with permission from the national committee overseeing animal experiments.

Establishing Modular Connections to the Intermediate Cerebellum in Rat

This series of experiments was aimed to chart the organization of climbing fiber projections to the intermediate cerebellum (paravermis) of the rat. These experiments made use of anterograde transport of iontophoretic injections with biotinylated dextran amine (BDA: Molecular Probes, Leiden, NL) that were aimed at selective areas of the rostral parts of both accessory olives, which are known to provide climbing fibers to the intermediate cerebellar cortex. In selected cases, small additional pressure injections were made in the contralateral cerebellar nuclei (aimed for the interposed nuclei) with a gold-lectin conjugate of bovine serum albumin with wheat germ agglutinate and $10 \mathrm{~nm}$ gold sol (Aurion, Wageningen, NL), which has been demonstrated to be an excellent retrograde tracer [22, 23]. After a survival time of 5-7 days, the animals were sacrificed, perfused, and the brain embedded in gelatin and serially and transversely sectioned at $40 \mu \mathrm{m}$. Selected serial sections were incubated with the $\mathrm{ABC}$ elite $^{\mathrm{TM}}$ technique (Vector Laboratories, Burlingame,

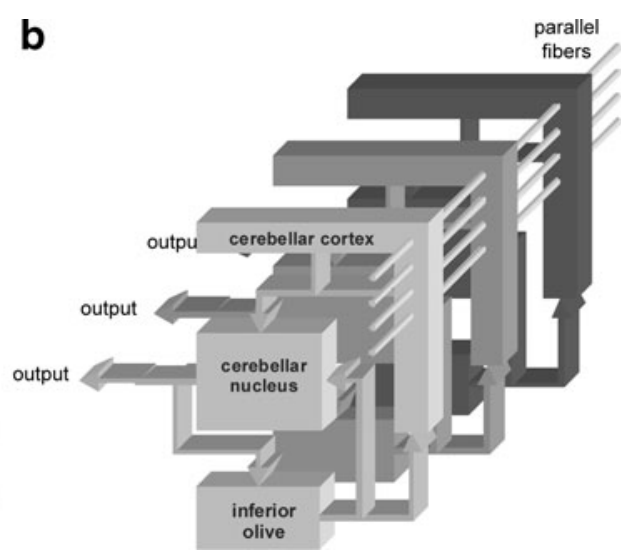

precerebellar mossy fiber systems such as the pontine nuclei, as well as to midbrain areas known to provide input to the inferior olive. b Diagram illustrating parallel organization of three cerebellar modules, each with their own specific output. Parallel fiber beams are included because they represent an obvious way of connecting modules. Modified from Ruigrok [59]

$\mathrm{CA}$ ) using either $\mathrm{DAB}$ or $\mathrm{DAB} /$ Cobalt for visualization of the tracer. Some of these series were selected for silver enhancement of the gold-lectin label (Aurion) or for zebrin II labeling (antibody kindly provided by Dr. Richard Hawkes, Calgary, Canada) using DAB visualization. In some series, triple labeling of BDA, gold-lectin, and zebrin II was obtained for light microscopical analysis. Further details are provided by Pijpers et al. [7].

\section{Relating Mossy Fiber Organization to Modular Patterns}

In order to demonstrate the longitudinal patterns of pontine mossy fiber projections to the cerebellar cortex in mice, small iontophoretic BDA injections were made in the basal pontine nuclei using a glass micropipette (Boele and Ruigrok, unpublished results). After 5-day survival, the animals were sacrificed, perfused, embedded in gelatin, and serially sectioned transversely at $40 \mu \mathrm{m}$. BDA was visualized as described above. Three-dimensional reconstruction was made with Neurolucida ${ }^{\mathrm{TM}}$ software (Microbrightfield, Colchester, VT) based on plots of one out of every two sections.

Other series of experiments make use of the excellent retrograde and anterograde transport characteristics of cholera toxin b subunit (CTb: List Biological Laboratories, Campbell, CA). In a first series of $\mathrm{CTb}$ experiments, iontophoretic injections were made in the paravermis of lobules VII and VIII of the rat cerebellum. After a survival time of 7 days, sections were incubated for CTb immunochemistry using $\mathrm{DAB} /$ Cobalt for visualization and double labeling with zebrin II (DAB). Evaluation of the injection site with respect to the zebrin II pattern in combination with the location of retrograde $\mathrm{CTb}$ labeling of neurons within 
the inferior olivary complex enabled accurate establishment of the zonal location of the injection site. The resulting collateral labeling of climbing fibers and mossy fiber rosettes was analyzed and quantified (mossy fiber rosettes) throughout the cerebellar cortex using Neurolucida ${ }^{\mathrm{TM}}$ software and entered in specially prepared diagrams of the unfolded and flattened cerebellar cortex [24]. Neurolucida $^{\mathrm{TM}}$ software was also used to prepare $3 \mathrm{D}$ diagrams of selected regions of the cerebellar cortex. These experiments were performed in collaboration with Dr. Richard Apps (University of Bristol, UK). More details are provided by Pijpers et al. [25].

In a follow-up series of experiments (Ruigrok, unpublished results), CTb was injected into the cerebellar nuclei, thereby, after the appropriate survival time, resulting in retrograde labeling of Purkinje cells as well as, by way of their nuclear collaterals, of olivary neurons and various mossy fiber sources. In addition, cerebellar cortical labeling was observed in climbing fiber collaterals and mossy fiber rosettes. The patterns of cortical labeling were visualized using Neurolucida ${ }^{\mathrm{TM}}$ software.

Modular Control of Individual Muscles: Retrograde Labeling with Rabies Virus

These experiments make use of the strictly retrograde and transneuronal route of infection by rabies virus. Between 5 and $30 \mu \mathrm{l}$ of the CVS 11 strain of rabies virus was injected into tibialis anterior or gastrocnemius muscle of the rat hind limb. This strain has been shown to be reliably infect synaptically connected neurons in a strictly retrograde direction without "leakage" of virus [26, 27]. After survival times of 4 to 6 days, the progression of the infection was monitored by sacrifice and perfusion of the rat, extraction of the spinal cord and brain, and subsequent serial sectioning and immunostaining with antirabies antibody. Selected series were also treated for zebrin II immunohistochemistry. 3D reconstructions were made using Neurolucida ${ }^{\mathrm{TM}}$. These experiments were performed in collaboration with Dr. Patrice Coulon (Université de la Méditerranée, Marseille, France). For further details, see Ruigrok et al. [28].

\section{Results}

\section{Modular Connectivity}

In establishing the detailed organization of the olivocortico-nuclear connections, the use of an independent reference frame provided by the Purkinje cell marker zebrin II has proven to be vital. This mouse monoclonal antibody zebrin II was prepared using immunization with a crude cerebellar homogenate of the weakly electric fish Apter- onotus $[29,30]$ and was later shown to be identical to aldolase $\mathrm{C}$ [31]. In rat, this antibody labels a midline strip of Purkinje cells and an additional five (rostral cerebellum) or six (caudal cerebellum) parasagittal strips of Purkinje cells, which are interspersed with strips of zebrin IInegative Purkinje cells. A central transverse region consisting mostly of vermal lobule $\mathrm{Vib} / \mathrm{c}$ and the associated hemispheral lobules of crus 1 , as well the caudalmost transverse region that consists of the ventral uvula (lobule IXc) and nodulus (lobule X) only contain zebrin II-positive Purkinje cells [2].

Figure 2a shows a standardized diagram of the unfolded and flattened right half of the cerebellar cortex in which the pattern of zebrin II labeling is shown. This diagram was constructed by following the continuation of the individual lobules throughout the serial sections of the cerebellum [7, $24,32]$. Although small differences in patterning may exist between individual animals, the general pattern has been demonstrated to be remarkably constant [11, 32].

In unraveling the zonal organization of olivo-cortical and cortico-nuclear projections by anterograde and retrograde neuroanatomical tracers, respectively, and by matching the resulting strips of anterogradely labeled climbing fibers (Fig. 2e1) and retrogradely labeled Purkinje cells with the zebrin II pattern, the organization of olivo-cortical and cortico-nuclear connections has been revealed in great detail as shown for the intermediate rat cerebellum in Fig. $2 b-d[7,8,11,32]$. It can be appreciated that the strips of climbing fibers resulting from a particular region of the inferior olivary complex can be followed over multiple lobules. In addition, several of these strips may be recognized in anterior and posterior cortical areas but are discontinuous in the central areas. In all instances, the strips beautifully adhere to patterning of the zebrin II. Combining injections of an anterograde tracer (BDA) in the inferior olive together with an injection of a retrograde tracer in the contralateral cerebellar nuclei enabled direct demonstration of the olivo-cortico-nuclear modular connectivity (Fig. 2e2) [7]. An overview of established cerebellar zones and their associated modules is shown in Table 1, which was based on data provided by references $[7,8,11,33]$ (also see [2]).

\section{Mossy Fibers and Cerebellar Modules}

The modular character of olivo-cortico-nuclear connections has been firmly established. It is interesting to see if and how the organization of the other main cerebellar afferent system, i.e., the mossy fibers, relates to this concept. Usually, mossy fiber organization has been referred to as patchy, revealing a crude somatotopy in which small, adjacent patches of cerebellar cortex respond to stimulation of nonadjacent areas of a particular body region, commonly referred to as fractured somatotopy [34, 35]. 

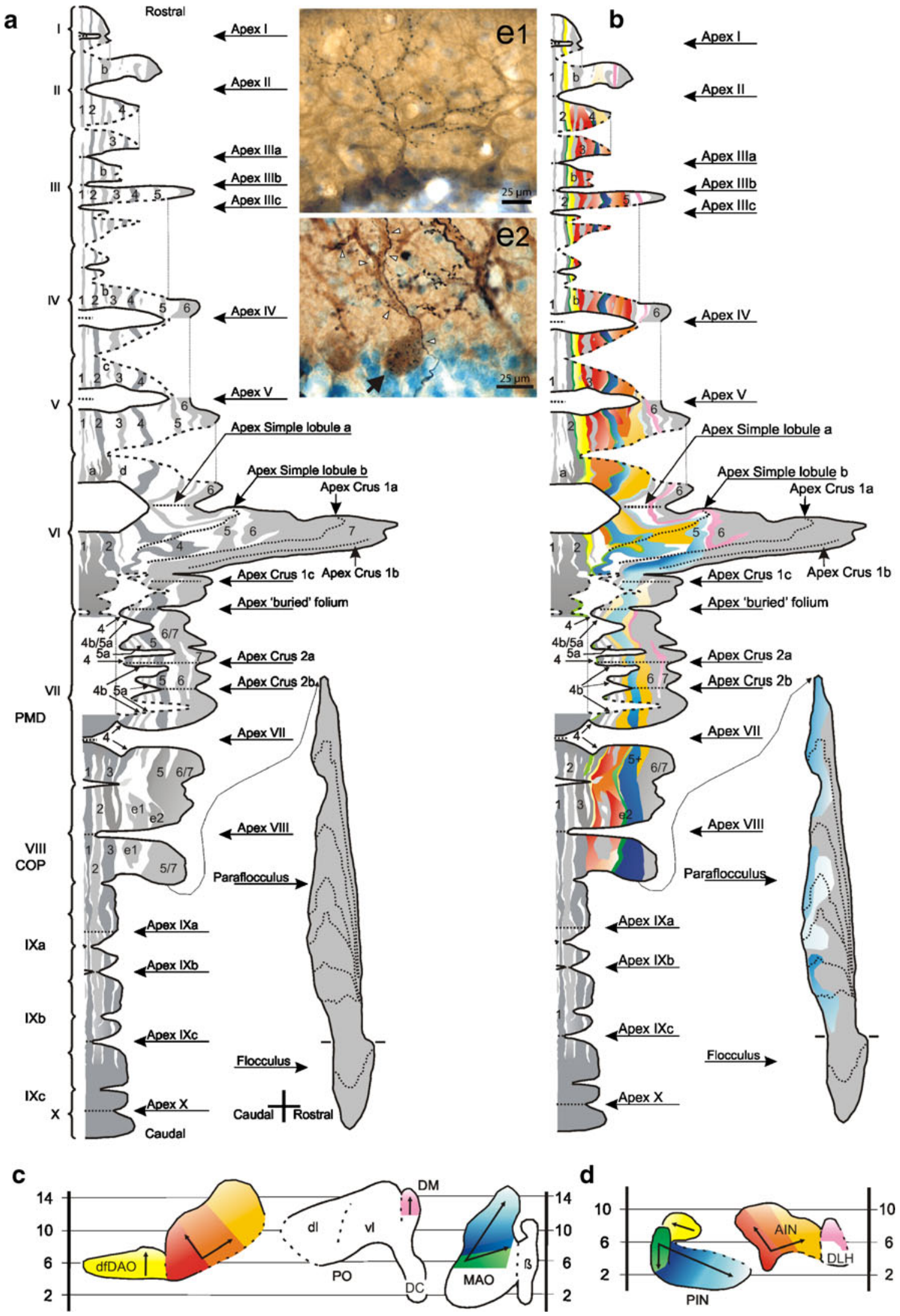
Fig. 2. a Diagram of the unfolded and flattened cerebellar cortex of the rat on which the zebrin II pattern has been indicated. b Similar diagram together with the longitudinal zonal climbing fiber pattern with colors that reflect the position of the BDA injections in a flattened reconstruction of the inferior olivary complex (c) and the related climbing fiber collateral labeling in a similarly prepared image of the flattened cerebellar nuclei (d). el Example of a BDA-labeled climbing fiber (black) terminating on the soma and dendrite of a zebrin II-positive Purkinje cell. e2 Example of a zebrin II-positive Purkinje cell (arrow), retrogradely labeled with gold-lectin after a small gold-lectin injection in the posterior interposed nucleus and innervated by a BDA-labeled climbing fiber resulting from a BDA injection into the rostral part of the medial accessory olive. Modified from Pijpers et al. [7]

However, this patchy functional organization is more difficult to recognize with anatomical techniques. Rather, recent reports have mostly stressed the longitudinal patterns of mossy fiber terminals that originate from a particular region [32, 36, 37]. Here, I show an example of the distribution of mossy fiber rosettes in the mouse cerebellar cortex after a small injection with the anterograde tracer biotinylated dextran amine (BDA) in the basilar pontine nuclei (Fig. 3a-c). Note that, in the $3 \mathrm{D}$ reconstruction, longitudinal patterns may be observed, but these do not stand out as clearly as the olivo-cortico-nuclear projections. Indeed, these patterns, at least in some cases, have been described to adhere to the overlying strip-like patterning of zebrin II [32, 38, 39]. However, the organization of projections from the basilar pontine nuclei in relation to the zebrin pattern has not yet been fully documented [40, 41]. To a large part, this is due to the complex intrinsic organization of the pontine nuclei and its in- and output characteristics (for review see [42]), which makes it virtually impossible to study the organization of the pontocerebellar projection in relation to the zebrin system using conventional anterograde tracing techniques.

Nevertheless, here, I like to report on two other types of experiments that lend further support to the notion that the organization of mossy fibers reveals aspects of a modular organization. These experiments make use of the specific tracing characteristics of the $b$ subunit of cholera toxin $(\mathrm{CTb})$. When injected in the posterior cortex of rats, this tracer is taken up by terminals of climbing fibers as well as of mossy fibers and subsequently retrogradely transported to their origin. However, eventually, CTb will also be transported anterogradely and therefore will also label all collaterals of these retrogradely labeled neurons [24, 43]. In this way, injections that are limited to a particular zone in the posterior cerebellum, e.g., C1 of lobule VIII, will not only result in retrogradely labeled olivary neurons in the lateral half of the ventral fold of the dorsal accessory olive $[7,18]$ but also in labeling of the collaterals of these climbing fibers that terminate in the anterior cerebellum $[25,43,44]$. Similarly, the neurons of origin of the mossy fibers terminating within the injection site will take up CTb, which will subsequently be distributed to their collaterals. When analyzing the distribution of climbing and mossy fiber collaterals, all labeled from a single cortical focus, it was noted that the climbing fiber collaterals are always accompanied by labeled mossy fiber collaterals but not vice versa (Fig. 3d-h) [24, 25, 43]. Strip-like fields of labeled mossy fiber rosettes are always found in more medial and/ or lateral regions of usually the same lobules. Interestingly, however, when collaterals of climbing fibers are found to terminate upon zebrin II-negative Purkinje cells, most noncorresponding mossy fiber collaterals are also noted below zebrin II-negative Purkinje cells and vice versa [25]. Supposing that zebrin II-negative or zebrin II-positive Purkinje cells form functionally distinct entities, this suggests that there may be a functional link in the distribution of all labeled mossy fiber collaterals from a particular focus.

In a similar type of experiment, $\mathrm{CTb}$ was injected in the cerebellar nuclei. In this way, collaterals of climbing fibers that terminate within the injected area of the cerebellar
Table 1 Overview of olivocortico-nuclear modules

Modules are referred to by its participating cortical zone

$A I N$ anterior interposed nucleus, $D A O$ dorsal accessory olive, $I C G$ interstitial cell groups, $L C N$ lateral cerebellar nucleus, $L V N$ lateral vestibular nucleus, $M A O$ medial accessory olive, MCN medial cerebellar nucleus, $P I N$ posterior interposed nucleus, $P O$ principal olivary nucleus

\begin{tabular}{lll}
\hline Inferior olive & Cortical zone & Cerebellar/vestibular nuclei \\
\hline Caudal MAO (central) & $\rightarrow \mathrm{A} 1 \rightarrow$ & MCN \\
Caudal MAO (lateral) & $\rightarrow \mathrm{Ax} \rightarrow$ & MCN/ICG \\
Intermediate MAO & $\rightarrow \mathrm{X} \rightarrow$ & ICG \\
Dorsal fold DAO & $\rightarrow \mathrm{B} \rightarrow$ & LVN \\
Caudal MAO (medial) & $\rightarrow \mathrm{A} 2 \rightarrow$ & Dorsolateral protuberance \\
Ventral fold DAO & $\rightarrow \mathrm{C} 1 \rightarrow$ & AIN \\
Intermediate MAO & $\rightarrow \mathrm{Cx} \rightarrow$ & ICG/PIN (medial) \\
Rostral MAO & $\rightarrow \mathrm{C} 2 \rightarrow$ & PIN \\
Ventral fold DAO & $\rightarrow \mathrm{C} 3 \rightarrow$ & AIN \\
Ventral leaf PO & $\rightarrow \mathrm{D} 1 \rightarrow$ & LCN (caudoventral) \\
Dorsomedial group PO & $\rightarrow \mathrm{D} 0 \rightarrow$ & Dorsolateral hump \\
Dorsal leaf PO & $\rightarrow \mathrm{D} 2 \rightarrow$ & LCN (rostrodorsal) \\
\hline
\end{tabular}




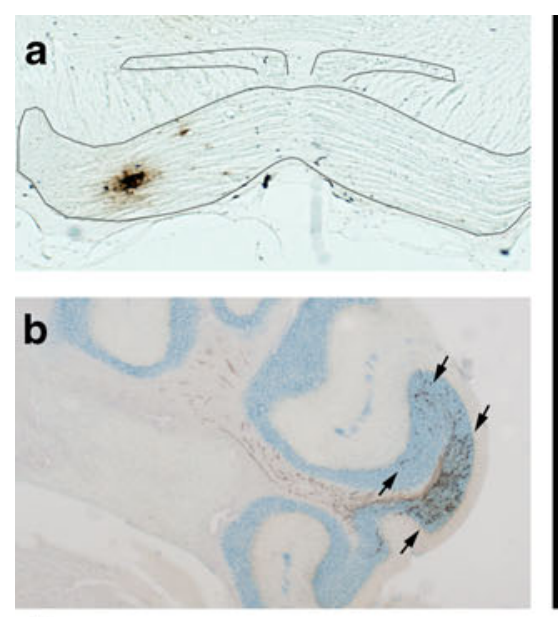

d

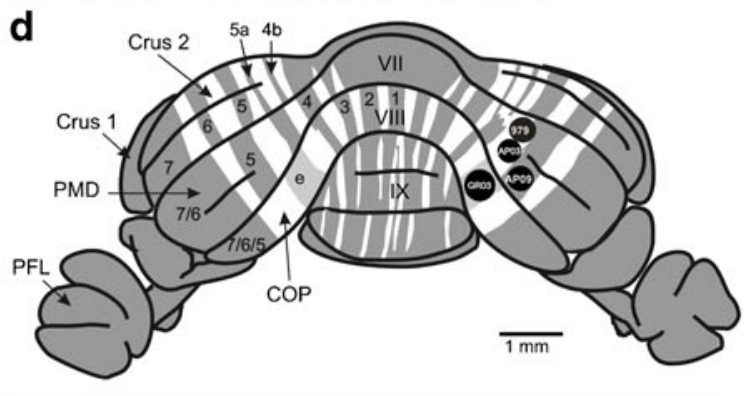

f Climbing fibers

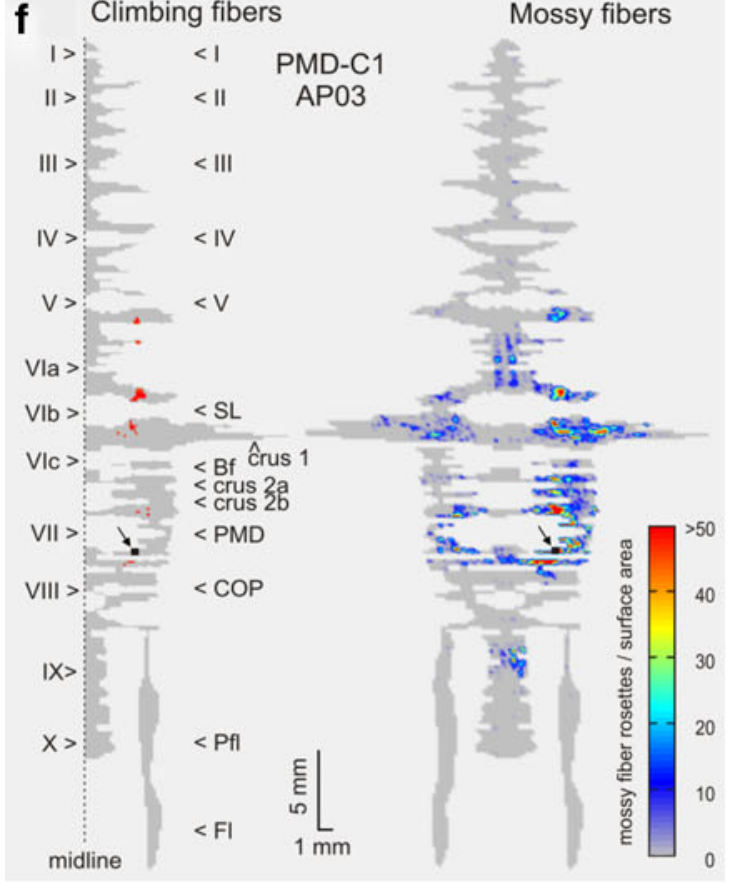

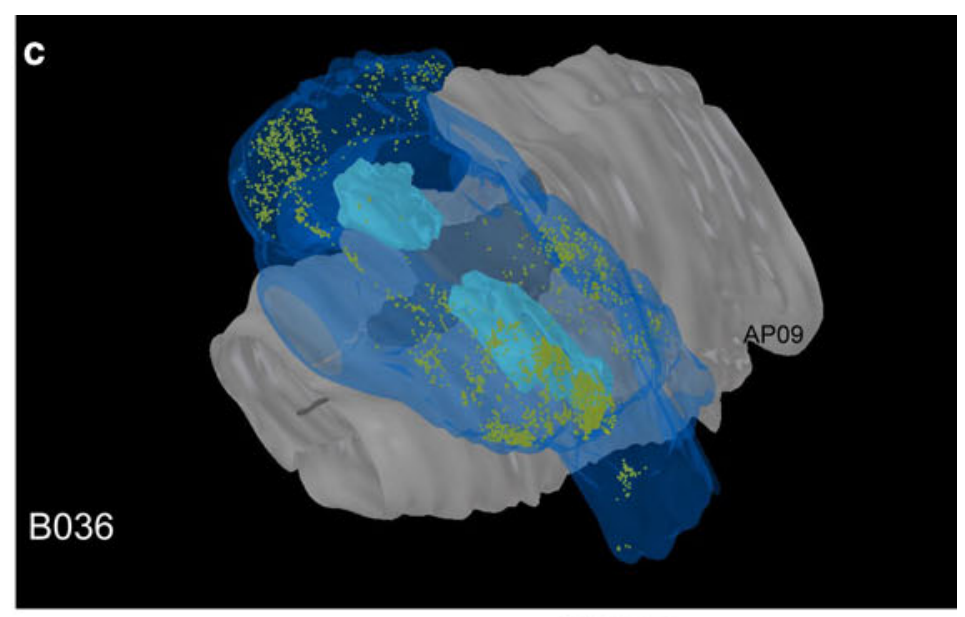

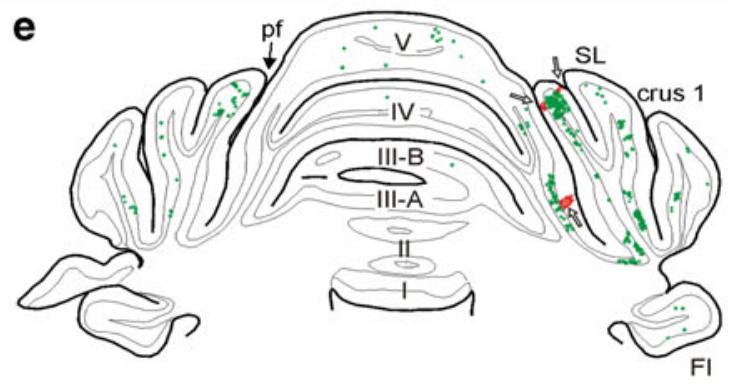

g Climbing fibers Mossy fibers

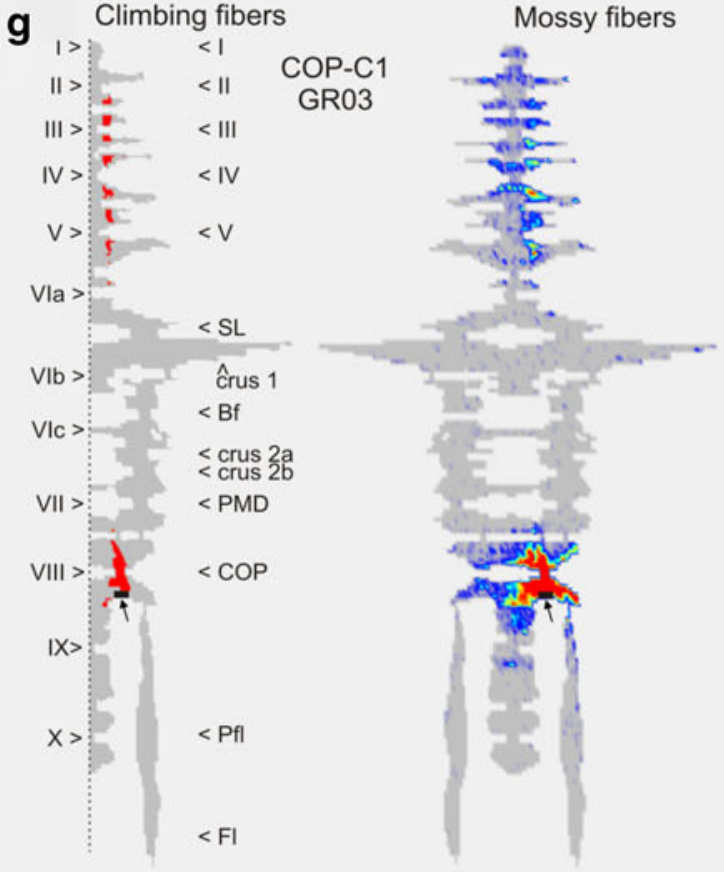

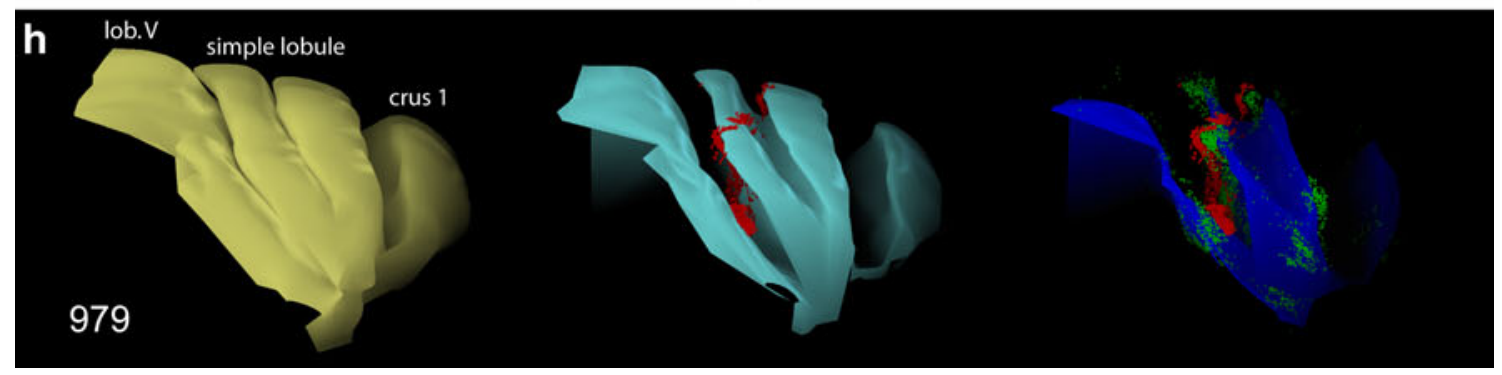


Fig. 3. Modular organization of mossy fiber projections. a Example of BDA injection in center of the left basal pontine nuclei of the mouse (B036). b Resulting labeling of mossy fiber rosettes in crus 2. Arrows point to two separate clusters of terminals. c $3 \mathrm{D}$ reconstruction based on plots of one out of every two sections showing the brainstem and transparent cerebellum (case B036). Labeled mossy fiber rosettes are indicated with yellow dots. In this view, approximately four longitudinal patterns of mossy fiber terminals can be appreciated at the right hand side of the posterior cerebellum. d Location of four CTb injections in the paravermis of lobules VII and VIII indicated in a standardized zebrin II representation of the caudal cerebellum. e Example of the resulting labeling in case AP09 in the anterior cerebellum. Red coloring indicates the position of terminal climbing fiber collaterals within the molecular layer, whereas green dots represent mossy fiber rosettes. Note that climbing fiber labeling is accompanied by dense labeling of mossy fiber rosettes. $\mathbf{f}$ Relation of resulting climbing fiber collateral labeling with that of mossy fiber collateral labeling in case AP03 with an injection into the $\mathrm{C} 1$ zone of lobule VII (paramedian lobule; PMD). Left hand panel shows position of labeled climbing fibers in a representation of the unfolded and flattened cerebellar cortex. Injection site is marked with an arrow. Right hand panel shows the distribution and density of mossy fiber terminals. Again, note that regions with climbing fiber labeling relate to high densities of labeled rosettes. $\mathbf{g}$ Similar to $\mathbf{f}$ for case GR03 with an injection in $\mathrm{C} 1$ of lobule VIII (COP copula). Note the difference in general lobular distribution of the labeling. $\mathbf{h} 3 \mathrm{D}$ reconstruction of resulting terminal climbing and mossy fiber labeling in case 979 where the injection was centered on C1 of PMD. Left hand panel shows the subpial surface of lobules V, simple lobule, and crus 1 (yellow). Middle panel shows the Purkinje cell layer (light blue) with labeled climbing fiber terminals (red). Left hand panel shows white matter contours with climbing fiber (red) and mossy fiber terminals (green). Note two strips of mossy fiber labeling, the middle of which accompanies the climbing fiber strip. Modified from Pijpers et al. [25]

nuclei will take up the tracer and will not only retrogradely label their parent neurons in the inferior olive but also their ongoing climbing fibers to the cortex. Comparable uptake by nuclear branches of mossy fiber collaterals will reveal the distribution of their origin as well as the distribution of their termination patterns in the cerebellar cortex. In addition, anterograde labeling of nucleocortical fibers as well as retrograde labeling of Purkinje cells will be observed. Within the distribution patterns of these highly different types of labeling, a remarkable overlap can be observed as shown for a single case in Fig. 4. Here, the $\mathrm{CTb}$ injection was centered on the central aspect of the anterior interposed nucleus (Fig. 4a), resulting in retrograde labeling of olivary neurons in the dorsal accessory olive (Fig. 4b). This injection, furthermore, resulted in labeling of characteristic strip-like climbing fiber labeling in the paravermis of lobules IV/V. These climbing fiber strips overlapped beautifully with the retrogradely labeled Purkinje cells as would be expected according to the concept of interconnected olivo-cortico-nuclear modules. However, in addition, the distribution of the mossy fiber rosettes also nicely corresponded to both the localization of labeled climbing fibers as well as labeled Purkinje cells (Fig. 4c-e). This suggests that not only the mossy fiber rosettes but also their nuclear collaterals distribute according to a rather precise patterning that corresponds to that of climbing fibers.

\section{Cerebellar Modules and Muscle Control}

Although the modular concept of cerebellar organization is generally well established by anatomical as well as physiological techniques [1,3], less information seems to be available on the way the individual modules contribute to coherent control of movement. Indeed, only for the control of the vestibulo-ocular reflex, the differential control of specific floccular zones or modules has been convincingly demonstrated [45].

In order to establish whether cerebellar modules may be involved in the control of individual muscles, we have injected rabies virus into either the tibialis anterior or gastrocnemius muscle of the rat hind limb [28]. Rabies virus is transported transneuronally in a strict retrograde fashion $[26,46]$. After an appropriate survival time (usually 5 days postinjection), Purkinje cells start to become infected in the cerebellar cortex. These Purkinje cells are arranged in beautiful strip-like patterns (Fig. 5a, b). Matching these patterns with the zebrin II pattern and from there with the related olivocerebellar zones indicated that these initial strips of rabies-infected Purkinje cells corresponded to the lateral $\mathrm{A} 1$ and the $\mathrm{B}$ zones $[2,8,11]$. Although the injected muscles are antagonists, there was a conspicuous overlap in large regions of the B zone (cf Fig. 5a2 and Fig. 5b2). However, interestingly, labeling within the lateral A1 zone was markedly different for both muscles. This zone revealed infected Purkinje cells mostly ipsilateral to the injected tibialis anterior, but labeled cells were noted mostly contralateral after injection of the gastrocnemius. Due to the diversity in labeled supraspinal structures, the precise route by which these Purkinje cells become infected cannot be established unequivocally from the present series of experiments. However, based on the timing of Purkinje cell infection, on the labeling patterns at earlier survival times, and on knowledge concerning anatomical connections, it seems highly likely that both the lateral A1 and B zone labeling reflect connections of the cerebellar cortex to the spinal cord by way of the lateral vestibular nucleus $[11,28]$. Increasing the survival time to 6 days resulted in labeling of many additional Purkinje cells which were all clustered to form clear longitudinal strips, which could be recognized throughout several lobules (Fig. 5c, d). These strips were observed in vermal and paravermal as well as hemispheral areas of the cerebellum, frequently demonstrating mirror images in both cerebellar halves. Rabies labeling in the cerebellar cortex reliably corresponded to labeling of the cerebellar nuclei and their targets such as the red nucleus and the ventromedial reticular formation at previous stages of the infection [28]. 

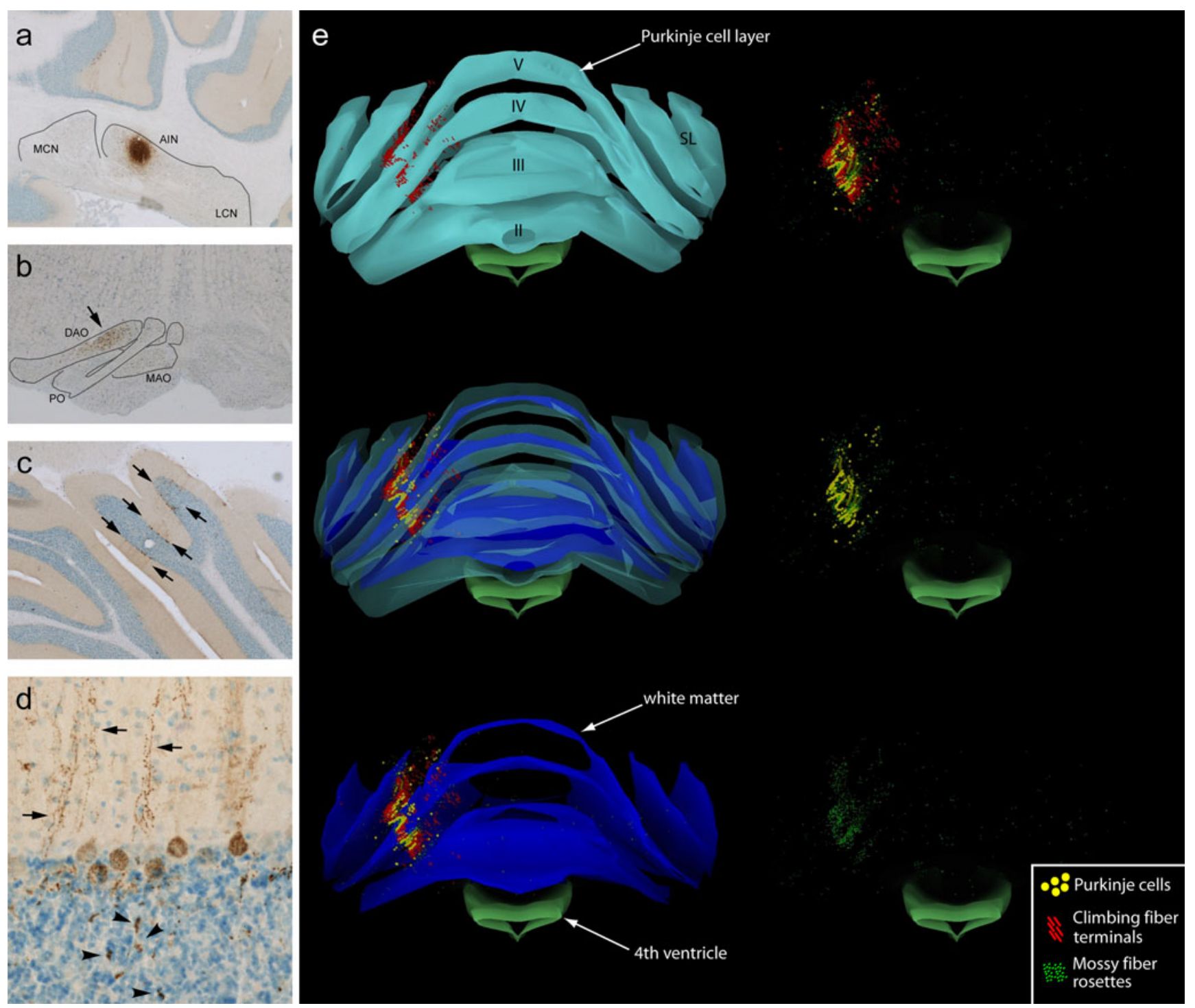

labeled climbing fibers (red). Middle panel shows transparent Purkinje

Fig. 4. Demonstration of modular organization of mossy fiber projection with nuclear injection of $\mathrm{CTb}$. a Injection of $\mathrm{CTb}$ in the anterior interposed nucleus (AIN). b Distribution of retrogradely labeled olivary neurons (arrow) was confined to the dorsal accessory olive (DAO). c Overview of cortical labeling in lobules IV and V indicating strip-like pattern of labeling (arrows). d Detail of labeling in lobule $\mathrm{V}$ where retrogradely labeled Purkinje cells are noted with overlying labeled climbing fibers (arrows) and underlying labeling of mossy fiber rosettes (arrowheads). e 3D representations of anterior lobe and simple lobule (SL) with fourth ventricle (green). Left hand upper panel shows Purkinje cell layer (light blue) with location of cell layer with labeled Purkinje cells (yellow) together with climbing fiber labeling (red). Bottom panel shows white matter contour (dark blue) together with mossy fiber terminal labeling (green), Purkinje cell labeling (yellow), and climbing fiber labeling (red). Labeling is seen without cerebellar contours in right hand panels (upper panel climbing and mossy fiber terminals and Purkinje cells; middle panel mossy fiber terminal labeling together with Purkinje cell labeling; bottom panel mossy fiber terminal labeling only). Note that all types of labeling clearly overlap in a parasagittally arranged strip in lobules $\mathrm{IV} / \mathrm{V}$ of the cerebellar cortex

\section{Discussion and Conclusions}

I have briefly reviewed some of our evidence on the modular organization of the cerebellum. Indeed, a long series of experiments in our lab and that of others (e.g., [ 7 , $8,47,48]$ ) has shown that Voogd's initial observation on the differential fiber composition of cerebellar white matter compartments [49] relates to a fundamental longitudinal organization of cortico-nuclear, olivo-cortical, and to some extent, also mossy fiber connections.

The participation of mossy fibers is particularly interesting in this respect. The specific matching of patterns of mossy fiber rosettes and climbing fiber terminals suggest that there is a special relation between granule cells and their overlying Purkinje cells and cortical interneurons. As such, the differential function of the ascending versus the 

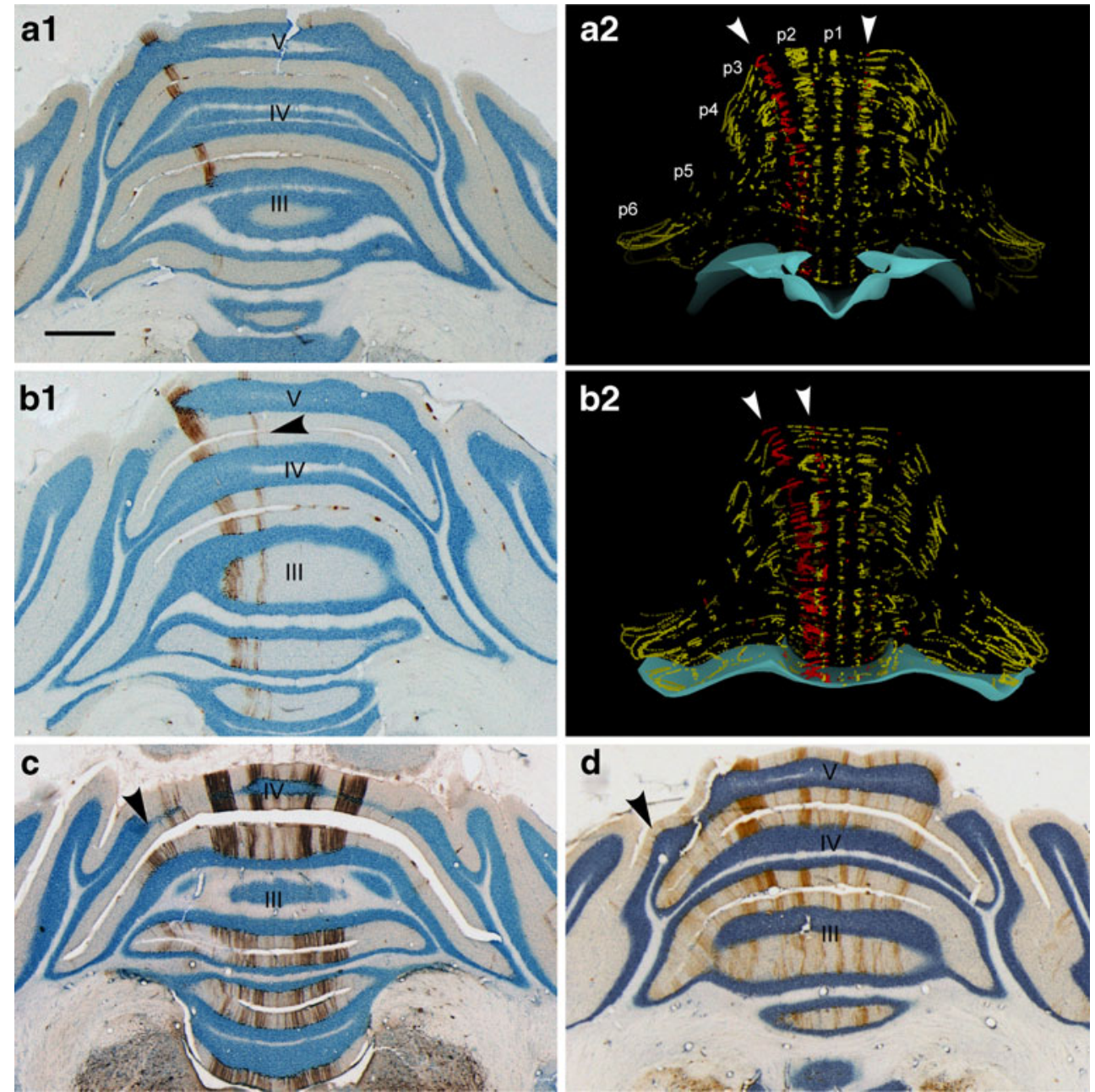

Fig. 5. Demonstration of involvement of multiple cerebellar modules in the control of single muscles using transneuronal retrograde transport of rabies virus in rat. al Pattern of Purkinje cell labeling in the lateral vermis 5 days after rabies injection in the ipsilateral gastrocnemius. $a 23 \mathrm{D}$ reconstruction of the anterior lobe of this case indicating rabies-labeled Purkinje cells (red) together with zebrin IIlabeled Purkinje cells (yellow). Note that the zebrin II-labeled bands identified as $\mathrm{p} 1-\mathrm{p} 6$ can all be recognized. The position of the rabieslabeled Purkinje cells between $\mathrm{p} 2$ and $\mathrm{p} 3$ (left hand white arrowhead) is identical to that of the B zone [11]. In addition, a contralateral strip of labeled Purkinje cells is noted just medial to the $\mathrm{p} 2$ zebrin II band

parallel part of the parallel fiber seems to be of particular importance [50-52]. Because either mossy fiber rosettes or parallel fibers also innervate cortical interneurons such as Golgi, basket, and stellate cells, longitudinal patterns have also been established with morphological [53] as well as physiological techniques [54]. On the other hand, the distribution of mossy fiber collaterals is clearly more widespread and encompasses several modules. As yet, it is not known to what extent these modules are functionally linked.

Because the modular output, by way of the different projections from the cerebellar nuclei, is directed to a (right hand white arrowhead), which corresponds to the location of the lateral A1 zone. b1, 2 Similar to $a 1,2$ after injection of rabies virus in the ipsilateral tibialis anterior muscle. This time both the B and lateral A1 zone (arrowhead) are noted ipsilateral to the injection. $c$ Pattern of infection 6 days after injection of the gastrocnemius muscle. Note that the original zones can still be recognized but also have a mirror representation in the other cerebellar half. However, several additional zones are also recognized (arrowhead) in paravermis and hemisphere (not shown). $d$ Similar to $c$ for injection of the tibialis anterior. Modified from Ruigrok et al. [28]

variety of regions in the brainstem and diencephalon [17], it is clear that modules exert an influence over a variety of different functions. For example, small injections in the anterior interposed nucleus result not only in terminal labeling within the red nucleus but also to inferior olive, pontine nuclei, and several thalamic nuclei. However, conversely, our rabies experiments show that multiple modules contribute to the control of specific muscles and that single modules may affect antagonistic muscles. This would suggest that modules are involved in the different aspects of motor control. Indeed, recent data making use of selective modular lesions resulting from cerebellar cortical 
injections with a suicide tracer suggest that the $\mathrm{C} 1$ hind limb module is particularly involved in gain control of reflexes during locomotion [55]. However, exactly how modular input by way of mossy and climbing fiber routes (e.g. [56]), in conjunction with the modular output, which may influence premotor areas in the midbrain as well as thalamic motor regions, in addition to projections to precerebellar regions such as basal pontine nuclei, reticular tegmental nucleus, and inferior olive [17, 57, 58], subserve cerebellar function is far from resolved.

Acknowledgments I would like to fondly and respectfully acknowledge Jan Voogd for his ongoing support, guidance, and encouragement throughout all of this work. Furthermore, I like to thank Dr. Richard Apps, Department of Physiology, Bristol University, UK and Dr. Patrice Coulon, Laboratoire Plasticité et Physio-Pathologie de la Motricité, Université de la Méditerranée, Marseille, for their passionate collaboration in some of the studies presented here. TR was supported by the Dutch Ministry of Health, Welfare, and Sports.

Open Access This article is distributed under the terms of the Creative Commons Attribution Noncommercial License which permits any noncommercial use, distribution, and reproduction in any medium, provided the original author(s) and source are credited.

\section{References}

1. Apps R, Garwicz M (2005) Anatomical and physiological foundations of cerebellar information processing. Nat Rev Neurosci 6(4):297-311

2. Apps R, Hawkes R (2009) Cerebellar cortical organization: a onemap hypothesis. Nat Rev Neurosci 10(9):670-681

3. Voogd J, Glickstein M (1998) The anatomy of the cerebellum. Trends Neurosci 2:305-371

4. Voogd J, Jaarsma D, Marani E (1996) The cerebellum: chemoarchitecture and anatomy. In: Swanson LW, Björklund A, Hökfelt T (eds) Handbook of chemical neuroanatomy: Elsevier Sience B. V. pp. 1-369

5. Groenewegen HJ, Voogd J (1977) Parasagittal zonation within the olivocerebellar projection. I. Climbing fiber distribution in the vermis of cat cerebellum. J Comp Neurol 174:417-488

6. Groenewegen HJ, Voogd J, Freedman SL (1979) The parasagittal zonation within the olivocerebellar projection. II. Climbing fiber distribution in the intermediate and hemispheric parts of cat cerebellum. J Comp Neurol 183:551-602

7. Pijpers A, Voogd J, Ruigrok TJ (2005) Topography of olivocortico-nuclear modules in the intermediate cerebellum of the rat. J Comp Neurol 492(2):193-213

8. Sugihara I, Shinoda Y (2004) Molecular, topographic, and functional organization of the cerebellar cortex: a study with combined aldolase C and olivocerebellar labeling. J Neurosci 24 (40):8771-8785

9. Sugihara I, Fujita H, Na J, Quy PN, Li BY, Ikeda D (2009) Projection of reconstructed single Purkinje cell axons in relation to the cortical and nuclear aldolase $\mathrm{C}$ compartments of the rat cerebellum. J Comp Neurol 512(2):282-304

10. Voogd J, Bigaré F (1980) Topographical distribution of olivary and cortico nuclear fibers in the cerebellum: a review. In: Courville J, de Montigny C, Lamarre Y (eds) The inferior olivary nucleus anatomy and physiology. Raven Press, New York, pp 207-234
11. Voogd J, Ruigrok TJ (2004) The organization of the corticonuclear and olivocerebellar climbing fiber projections to the rat cerebellar vermis: the congruence of projection zones and the zebrin pattern. J Neurocytol 33(1):5-21

12. De Zeeuw CI, Holstege JC, Ruigrok TJH, Voogd J (1989) Ultrastructural study of the GABAergic, cerebellar and mesodiencephalic innervation of the cat medial accessory olive: anterograde tracing combined with immunocytochemistry. J Comp Neurol 284:12-35

13. Ruigrok TJ, Voogd J (2000) Organization of projections from the inferior olive to the cerebellar nuclei in the rat. J Comp Neurol 426(2):209-228

14. Ruigrok TJH, Voogd J (1990) Cerebellar nucleo-olivary projections in rat. An anterograde tracing study with Phaseolus vulgarisleucoagglutinin (PHA-L). J Comp Neurol 298:315-333

15. van der Want JJL, Wiklund L, Guegan M, Ruigrok T, Voogd J (1989) Anterograde tracing of the rat olivocerebellar system with Phaseolus vulgaris-leucoagglutinin (PHA-L). Demonstration of climbing fiber collateral innervation of the cerebellar nuclei. $\mathrm{J}$ Comp Neurol 288:1-18

16. Chan-Palay V (1977) Cerebellar dentate nucleus: organization, cytology and transmitters. Springer, Berlin

17. Teune TM, van der Burg J, van der Moer J, Voogd J, Ruigrok TJH (2000) Topography of cerebellar nuclear projections to the brain stem in the rat. In: Gerrits NM, Ruigrok TJH, De Zeeuw CI (eds) Cerebellar modules: molecules, morphology and function. Elsevier Science B.V, Amsterdam, pp 141-172

18. Atkins MJ, Apps R (1997) Somatotopical organisation within the climbing fibre projection to the paramedian lobule and copula pyramidis of the rat cerebellum. J Comp Neurol 389:249-263

19. Ekerot CF, Larson B (1979) The dorsal spino-olivocerebellar system in the cat. II. Somatotopical organization. Exp Brain Res 36(2):219-232

20. Oscarsson O (1980) Functional organization of olivary projections to the cerebellar anterior lobe. In: Courville J, de Montigny C, Lamarre Y (eds) The inferior olivary nucleus, anatomy and physiology. Raven Press, New York, pp 279-289

21. Oscarsson O, Sjolund B (1977) The ventral spino-olivocerebellar system in the cat. I. Identification of five paths and their termination in the cerebellar anterior lobe. Exp Brain Res 28(5):469-486

22. Ruigrok TJ, Apps R (2007) A light microscope-based double retrograde tracer strategy to chart central neuronal connections. Nat Protoc 2(8):1869-1878

23. Ruigrok TJH, Teune TM, van der Burg J, Sabel-Goedknegt $H$ (1995) A retrograde double labeling technique for light microscopy. A combination of axonal transport of cholera toxin Bsubunit and a gold-lectin conjugate. J Neurosci Meth 61:127-138

24. Ruigrok TJ (2003) Collateralization of climbing and mossy fibers projecting to the nodulus and flocculus of the rat cerebellum. J Comp Neurol 466(2):278-298

25. Pijpers A, Apps R, Pardoe J, Voogd J, Ruigrok TJ (2006) Precise spatial relationships between mossy fibers and climbing fibers in rat cerebellar cortical zones. J Neurosci 26(46):12067-12080

26. Kelly RM, Strick PL (2000) Rabies as a transneuronal tracer of circuits in the central nervous system. J Neurosci Methods 103 (1):63-71

27. Ugolini G (2008) Use of rabies virus as a transneuronal tracer of neuronal connections: implications for the understanding of rabies pathogenesis. Dev Biol (Basel) 131:493-506

28. Ruigrok TJ, Pijpers A, Goedknegt-Sabel E, Coulon P (2008) Multiple cerebellar zones are involved in the control of individual muscles: a retrograde transneuronal tracing study with rabies virus in the rat. Eur J Neurosci 28(1):181-200

29. Leclerc N, Schwarting GA, Herrup K, Hawkes R (1992) Compartmentation in mammalian cerebellum: zebrin II and Ppath antibodies define three classes of sagittally organized bands of Purkinje cells. Proc Natl Acad Sci USA 89:5006-5010 
30. Brochu G, Maler L, Hawkes R (1990) Zebrin II: a polypeptide antigen expressed selectively by Purkinje cells reveals compartments in rat and fish cerebellum. J Comp Neurol 291:538-552

31. Ahn AH, Dziennis S, Hawkes R, Herrup K (1994) The cloning of zebrin II reveals its identity with aldolase C. Development 120:20812090

32. Voogd J, Ruigrok TJH (1997) Transverse and longitudinal patterns in the mammalian cerebellum. In: De Zeeuw CI, Strata P, Voogd J (eds) The cerebellum: from structure to control. Elsevier, Amsterdam, pp 21-37

33. Sugihara I, Shinoda Y (2007) Molecular, topographic, and functional organization of the cerebellar nuclei: analysis by three-dimensional mapping of the olivonuclear projection and aldolase C labeling. J Neurosci 27(36):9696-9710

34. Shambes GM, Beermann DH, Welker W (1978) Multiple tactile areas in cerebellar cortex: another patchy cutaneous projection to granule cell columns in rats. Brain Res 157:123-128

35. Welker W (1987) Spatial organization of somatosensory projections to granule cell cerebellar cortex: functional and connectional implications of fractured somatotopy (summary of Wisconsin studies). In: King JS (ed) New concepts in cerebellar neurobiology. Alan R. Liss, Inc, New York, pp 239-280

36. Serapide MF, Panto MR, Parenti R, Zappala A, Cicirata F (2001) Multiple zonal projections of the basilar pontine nuclei to the cerebellar cortex of the rat. J Comp Neurol 430(4):471-484

37. Wu HS, Sugihara I, Shinoda Y (1999) Projection patterns of single mossy fibers originating from the lateral reticular nucleus in the rat cerebellar cortex and nuclei. J Comp Neurol 411(1):97118

38. Ji Z, Hawkes R (1994) Topography of Purkinje cell compartments and mossy fiber terminal fields in lobules II and III of the rat cerebellar cortex: spinocerebellar and cuneocerebellar projections. Neuroscience 61:935-954

39. Ruigrok TJH, Cella F (1995) Precerebellar nuclei and red nucleus. In: Paxinos G (ed) The rat nervous system, 2nd edn. Academic, Sydney, pp 277-308

40. Odeh F, Ackerley R, Bjaalie JG, Apps R (2005) Pontine maps linking somatosensory and cerebellar cortices are in register with climbing fiber somatotopy. J Neurosci 25(24):5680-5690

41. Pijpers A, Ruigrok TJ (2006) Organization of pontocerebellar projections to identified climbing fiber zones in the rat. J Comp Neurol 496(4):513-528

42. Ruigrok TJH (2004) Precerebellar nuclei and red nucleus. In: Paxinos G (ed) The rat nervous system, 3rd edn. Elsevier Academic Press, San Diego, pp 167-204

43. Voogd J, Pardoe J, Ruigrok TJ, Apps R (2003) The distribution of climbing and mossy fiber collateral branches from the copula pyramidis and the paramedian lobule: congruence of climbing fiber cortical zones and the pattern of zebrin banding within the rat cerebellum. J Neurosci 23(11):4645-4656

44. Sugihara I, Wu HS, Shinoda Y (2001) The entire trajectories of single olivocerebellar axons in the cerebellar cortex and their contribution to cerebellar compartmentalization. J Neurosci 21 (19):7715-7723

45. van der Steen J, Simpson JI, Tan J (1991) Representation of threedimensional eye movements in the cerebellar flocculus of the rabbit. In: Schmid R, Zambarbieri D (eds) Oculomotor control and cognitive processes. Elsevier Science Publishers B.V, North Holland, pp 63-77

46. Ugolini G (1995) Specificity of rabies virus as a transneuronal tracer of motor networks: Transfer from hypoglossal motoneurons to connected second-order and higher order central nervous system cell groups. J Comp Neurol 356:457-480

47. Buisseret-Delmas C, Angaut P (1993) The cerebellar olivocortico-nuclear connections in the rat. Progr Neurobiol 40:63-87

48. Ruigrok TJH (1997) Cerebellar nuclei: the olivary connection. In: De Zeeuw CI, Strata P, Voogd J (eds) The cerebellum: from structure to control. Elsevier Science B.V, Amsterdam, pp 162-197

49. Voogd J (1964) The cerebellum of the cat: structure and fiber connections. Van Gorcum, Assen

50. Gundappa-Sulur G, De Schutter E, Bower JM (1999) Ascending granule cell axon: an important component of cerebellar cortical circuitry. J Comp Neurol 408(4):580-596

51. Llinas R (1982). Radial connectivity in the cerebellar cortex: a novel view regarding the functional organization of the molecular layer. Exp Brain Res (suppl 6):189

52. Sims P, Hartell NA (2006) Differential susceptibility to synaptic plasticity reveals a functional specialization of ascending axon and parallel fiber synapses to cerebellar Purkinje cells. J Neurosci 26 (19):5153-5159

53. Sillitoe RV, Chung SH, Fritschy JM, Hoy M, Hawkes R (2008) Golgi cell dendrites are restricted by Purkinje cell stripe boundaries in the adult mouse cerebellar cortex. J Neurosci 28 (11):2820-2826

54. Gao W, Chen G, Reinert KC, Ebner TJ (2006) Cerebellar cortical molecular layer inhibition is organized in parasagittal zones. $\mathrm{J}$ Neurosci 26(32):8377-8387

55. Pijpers A, Winkelman BH, Bronsing R, Ruigrok TJ (2008) Selective impairment of the cerebellar $\mathrm{C} 1$ module involved in rat hind limb control reduces step-dependent modulation of cutaneous reflexes. J Neurosci 28(9):2179-2189

56. Apps R (2000) Gating of climbing fibre input to cerebellar cortical zones. Prog Brain Res 124:201-211

57. Teune TM, van der Burg J, De Zeeuw CI, Voogd J, Ruigrok TJH (1998) Single Purkinje cell can innervate multiple classes of projection neurons in the cerebellar nuclei of the rat: a light microscopic and ultrastructural triple-tracer study in the rat. J Comp Neurol 392:164-178

58. Teune TM, Van der Burg J, Ruigrok TJH (1995) Cerebellar projections to the red nucleus and inferior olive originate from separate populations of neurons in the rat. A non-fluorescent double labeling study. Brain Res 673:313-319

59. Ruigrok TJH (2007) Role of the cerebellum. In: Wolters EC, Laar TV, Berendse HW (eds) Parkinsonism and related disorders. VU University Press, Amsterdam 